\title{
Newly Formed Nail from the Remnant Germinal Matrix after Reconstruction of the Amputated Fingertip: A Review of Two Cases
}

\author{
Jung Hwan Um (1D, Soon Heum Kim (D), Dong In Jo (1) \\ Department of Plastic and Reconstructive Surgery, Konkuk University Chungju Hospital, Konkuk University School of Medicine, Chungju, Korea
}

\begin{abstract}
Meticulous debridement before reconstructive surgery for fingertip amputation, one of the most common hand injuries, is often crucial for better prognoses. Complete debridement to prevent abnormal formation of the nail plate will result in better quality of life for patients with a small remnant germinal matrix. The author will report two cases where nail plates formed abnormally after reconstruction of an amputated fingertip.
\end{abstract}

Keywords: Fingers; Amputation; Nail; Germinal matrix

\section{Introduction}

Fingertips are more likely to be damaged than other parts of the body, because they are constantly in use and in contact with surfaces [1]. Because fingertip amputation limits the function of the finger, it is necessary to perform reconstruction to restore the finger as much as possible. Unfortunately, in many cases of amputation, the margin of the damaged finger is often crushed, jagged and not clean. In these cases, the soft tissue responsible for the growth of the nail plate, namely the nail bed and the germinal matrix, are likely to be injured as well. If the remnant nail bed or germinal matrix is small, complete debridement to prevent new formation of the nail plate is preferred for better quality of life for the patient [2]. Otherwise, the nail plate may grow abnormally in the residual germinal matrix as a mass-like lesion, causing complications such as abnormal sensation including cold intolerance, focal pain, and infections $[3,4]$. The author reports two cases in which the nail plate was newly formed after the reconstruction was performed. This study was approved by the Institutional Review Board (IRB) of Konkuk University Chungju Hospital (IRB No. KUCH 202004-011). The patients provided written informed consent for the publication and the use of their images.

\section{Cases}

Case 1

A 59-year-old male patient with underlying diseases of diabetes mellitus and arrhythmia had come to the emergency room. The distal end of his left second finger was amputated after being pressed into a heavy machine (Fig. 1). Because the patient did
Case Report

Received: July 30, 2020

Revised: October 5, 2020

Accepted: October 5, 2020

Corresponding author:

Dong In Jo, M.D., Ph.D.

Department of Plastic and Reconstructive Surgery, Konkuk University Chungju Hospital, Konkuk University School of Medicine, 82 Gugwon-daero, Chungju 27376, Korea Tel: +82-43-840-8860

Fax: +82-43-840-9862

E-mail: cozarmd@kku.ac.kr

This is an Open Access article distributed under the terms of the Creative Commons Attribution Non-Commercial License (https://creativecommons.org/licenses/by-nc/4.0/) which permits unrestricted non-commercial use, distribution, and reproduction in any medium, provided the original work is properly cited.

(c) 2020 Korean Wound Management Society 

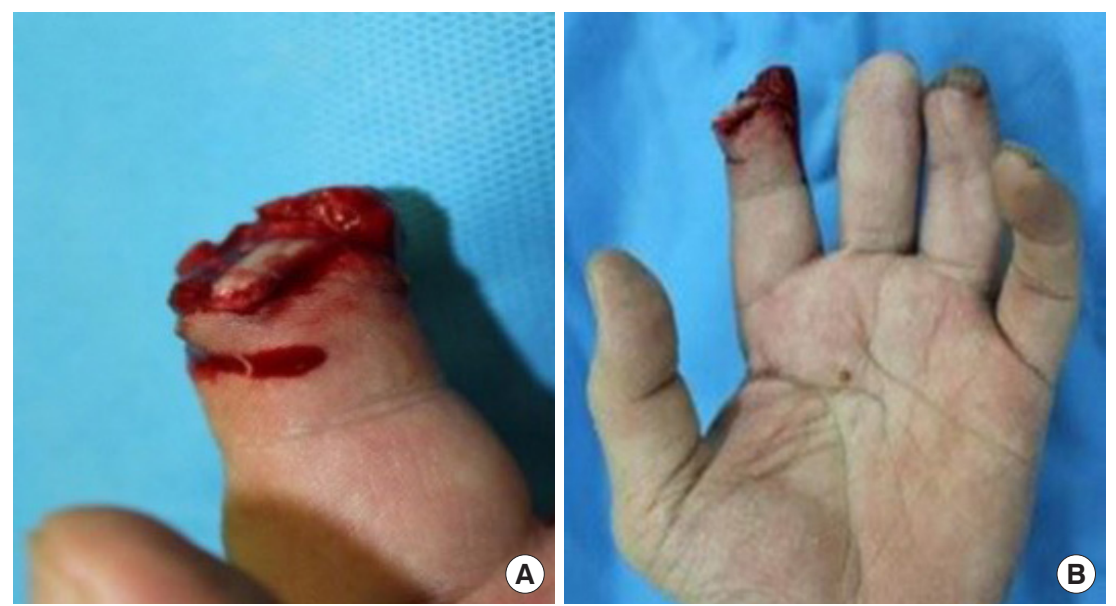

Fig. 1. Clinical photographs before surgery of case 1 . The left second fingertip was amputated after being pressed into a heavy machine. Detailed finger view (A) and full view (B).
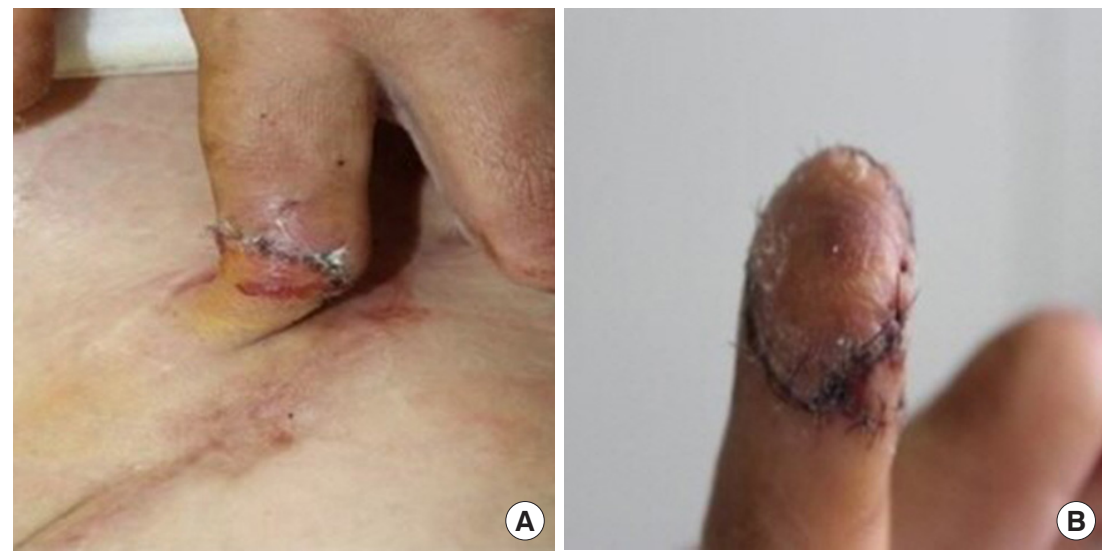

Fig. 2. Clinical photographs after surgery of case 1. (A) Because the patient did not bring the amputated segment, groin flap was performed: dorsal view. (B) Flap detachment and local advancement flap surgery were performed: palmar view.

not bring the amputated segment at that time, replantation could not be considered. Thus the same day, groin flap was performed instead of stump revision, to minimize distal phalanx length reduction (Fig. 2A). Wide and meticulous debridement was also performed, as the distal end of the amputated finger was observed to have a crushed and tattered margin. The base of the distal phalanx was preserved, and the insertion site of the second extensor digitorum communis tendon in the distal phalanx was also intact. The motor function of the second distal interphalangeal joint was preserved as well. Because the nail bed was totally amputated, all visible remnant germinal matrix was removed. Massive irrigation and empirical antibiotics were administered as usual, and the involved digit remained stable without complications such as infection, hematoma, or skin necrosis.

The patient was discharged on postoperative day (POD) 7 , and flap detachment and local advancement flap were performed on POD14, which remained stable afterward without complication (Fig. 2B).

Eight years after initial treatment, he presented with a chief complaint of a hard mass on the dorsal side of the fingertip on the left second finger (Fig. 3). The mass was elevated and keratinized, with a size of about 3-4 mm. No pain, tenderness, or discharge was observed. A mass was first detected three to 4 months after the surgery. At that time, it measured about a grain of rice (less than $1 \mathrm{~mm}$ ), and erythema and local heating were observed. The mass became harder, and the patient removed it himself with a toothpick. No additional discharge or erythema was observed in the removed lesion, but after 1 month, the mass grew back to the same size. After that, it was removed four additional times, but it recurred every time. Due to personal reasons, the patient had not been able to visit the 

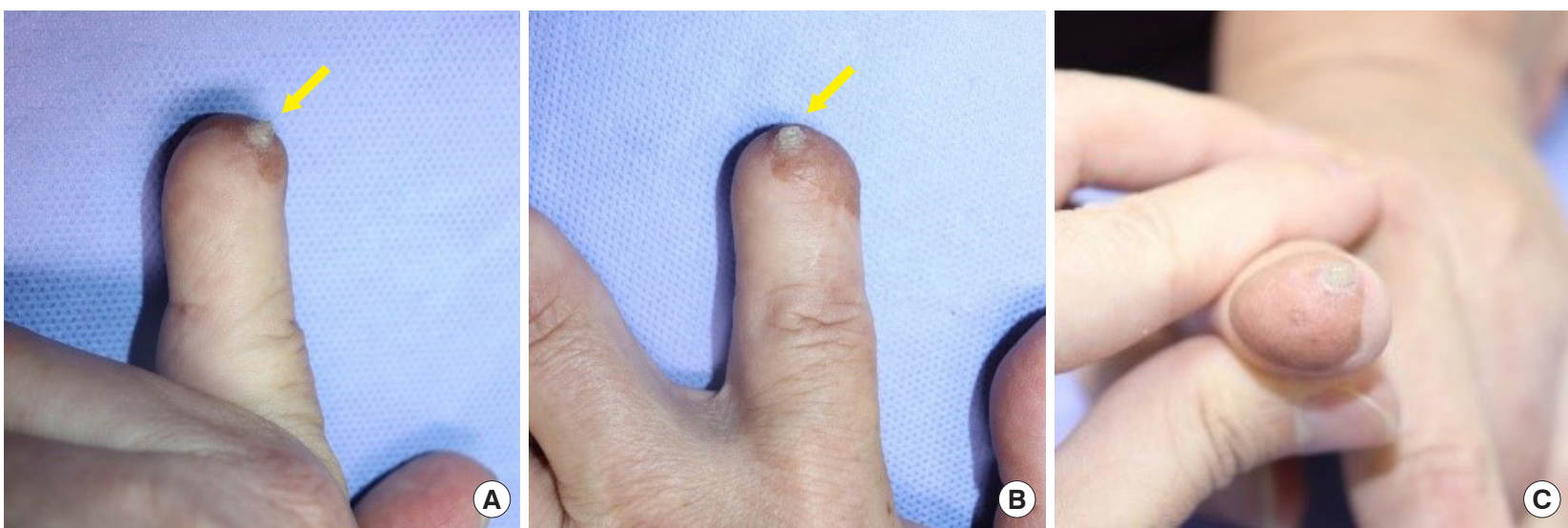

Fig. 3. Photographs 8 years after surgery of case 1 . The patient presented with a mass on the dorsal side of the fingertip. A hard mass (yellow arrow) in the involved digit was observed. (A) Ulnar view. (B) Dorsal view. (C) Tip view.
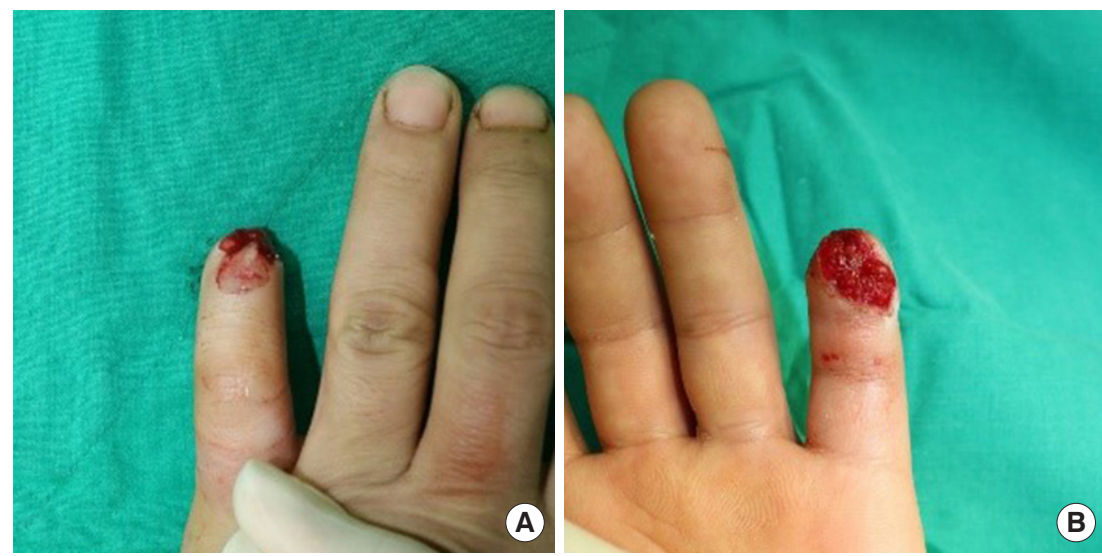

Fig. 4. Photographs before surgery of case 2. The left fifth fingertip was amputated after being buried between heavy iron plates. (A) Dorsal view. (B) Palmar view.

hospital for 8 years, and he had been removing the mass using a nail clipper every 2 weeks. The patient underwent an excisional biopsy under local anesthesia. The results of the biopsy were reported as nail tissue.

\section{Case 2}

A 38-year-old male patient with no underlying disease had come to the emergency room. His left fifth fingertip was amputated after being buried between heavy iron plates (Fig. 4). Replantation was impossible because the patient did not bring the amputated segment. On posttraumatic day (PTD) 1, wide and meticulous debridement was performed because the distal end of the amputated finger had a crushed and tattered margin. Since the remnant matrix was very small, all were removed to prevent the formation of deformed nails. To promote the growth of granulation tissue, vacuum-assisted closure was also applied.

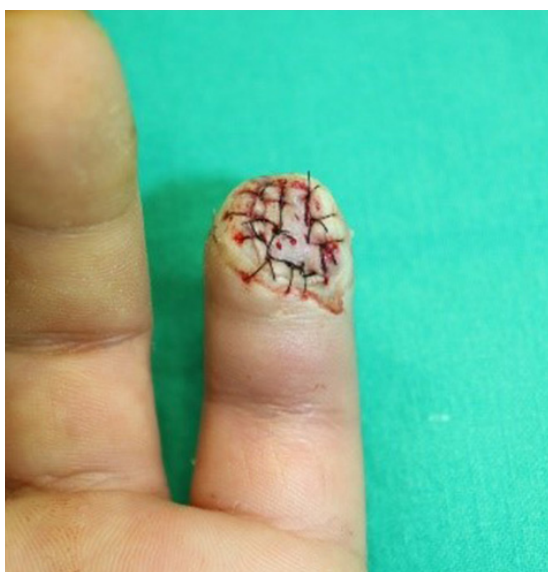

Fig. 5. A clinical photograph after surgery of case 2. A full-thickness skin graft surgery was performed.

Full-thickness skin graft was performed on PTD11 by harvesting the skin from the ipsilateral forearm (Fig. 5). There 

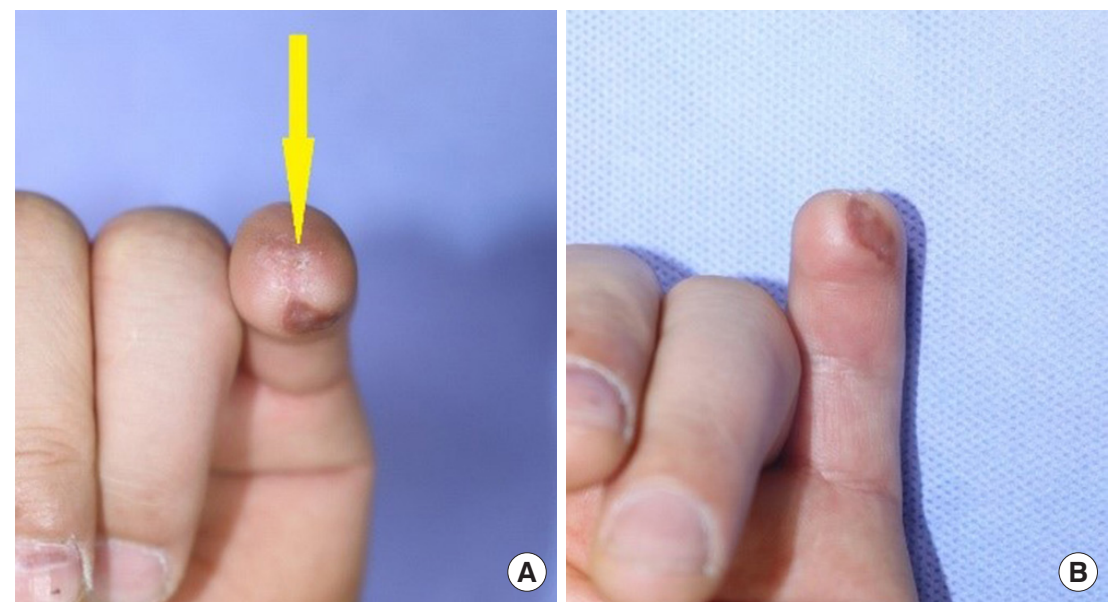

Fig. 6. Clinical photographs 2 months after surgery of case 2. A mass (yellow arrow) on dorsal side of the fingertip was observed. (A) Tip view. (B) Palmar view.

were no complications such as infection, hematoma, or skin necrosis. All stitches were removed on POD11.

Two months after initial treatment, he presented with a chief complaint of a hard mass on the dorsal side of the fingertip of the left fifth finger (Fig. 6). No lesion was grossly observed, and when the area was touched, a focal site of about $1 \times 1 \mathrm{~mm}$ was firmly palpable. There were no findings of discharge or erythema. The patient underwent excision under local anesthesia. Biopsy was not performed because the mass was smaller than the minimum size for performing biopsy to discriminate the nail plate [5]. However, the mass was located on the dorsum of the finger, in the same location as the removed germinal matrix, and the rigid, keratinized translucent features of the mass corresponded to the clinical features of the nail plate. For this reason, the removed mass was thought to be an abnormal nail from the remnant germinal matrix.

\section{Discussion}

The nail is one of the structures that make up the fingertip, and although small, it serves many functions in finger movement. First, the nail protects the distal phalanx, fingertip, and surrounding soft tissues from injuries [6]. Second, it detects the counter pressure of the finger pulp and enables precise, delicate movements. Third, the nail increases the sensitivity of the fingertip by sensing the counter force when the fingertip touches an object. Finally, it functions as a tool for performing extended precision grip and cutting or scraping actions.

As seen above, the nail is a structure that plays a substantial role in the function of the finger. For this reason, a criterion on the extent of injury to the nail that justifies complete excision of the germinal matrix is needed. Literature suggests that when more than the proximal $1 / 2$ of the nail is invaded, the risk of complications such as hook nails and nail remnants is increased, also increasing the need for secondary surgery. Therefore, in this case, complete removal of the germinal matrix is required $[7,8]$.

If there is no residual nail after fingertip injury, both function and appearance of the finger is damaged substantially, and compensation is required. One option is to perform nail reconstruction by grafting a part of the nail bed of an uninjured finger or toe, and another is to apply patient-specific finger prosthetics including the nail using three-dimensional printing technology. These are ways that a finger that has lost a nail can be functionally and cosmetically restored $[9,10]$.

According to literature review, reports of new nail plate formation after reconstructive surgery for an amputated fingertip are rare [11]. It is believed that is because the patients often dismiss the plate formation as a mild symptom and they remove the newly formed nail plate themselves. Amputated fingertips rarely have sharp and clear margins. Instead, the margins are often crushed and tattered because they are pressed by heavy objects or caught in rotating machines. It is clear that this marginal difference is an important factor for the prognosis in cases of replantation [12]. It is also important for reconstructive procedures to be performed when there is no amputated segment. Physicians should also be aware of the importance of massive irrigation and meticulous debridement before stump revision. In addition, as the germinal matrix is the productive organ of the nail plate, even if just a little tissue is 
left, an abnormal nail plate might grow to resemble a mass-like lesion, as in our cases. Because it does not function as a healthy nail plate, an abnormal plate can cause patient discomfort, including foreign-body sensation, and it can catch on clothing, as experienced by our patients.

As in the case above, if the nail plate grows into the skin, it is easy to remove, but if a cyst or hamartoma occurs covered by soft tissue, it becomes difficult to remove, and more severe complications can arise. To improve prognosis, clinicians should keep in mind the importance of wide and meticulous debridement.

\section{Conflict of interest}

No potential conflict of interest relevant to this article was reported.

\section{ORCID iDs}

Jung Hwan Um

Soon Heum Kim

Dong In Jo

https://orcid.org/0000-0002-9129-5582

https://orcid.org/0000-0001-9773-4753

https://orcid.org/0000-0002-3075-4482

\section{References}

1. Lemmon JA, Janis JE, Rohrich RJ. Soft-tissue injuries of the fingertip: methods of evaluation and treatment: an algorithmic approach. Plast Reconstr Surg 2008;122:105e-117e.

2. Lee DH, Mignemi ME, Crosby SN. Fingertip injuries: an update on management. J Am Acad Orthop Surg 2013;21: 756-66.

3. Golinvaux NS, Maslow JI, Hovis JP, et al. Fingertip injury and management. JBJS Essent Surg Tech 2019;9:e30.

4. Li J, Guo Z, Zhu Q, et al. Fingertip replantation: determinants of survival. Plast Reconstr Surg 2008;122:833-9.

5. Grover C, Bansal S. Nail biopsy: a user's manual. Indian Dermatol Online J 2018;9:3-15.

6. Onumah N. Nail surgery [Internet]. New York: Medscape [cited 2020 Oct 23]. Available from: https://emedicine. medscape.com/article/1126725-overview.

7. Champagne L, Hustedt JW, Walker R, et al. Digital tip amputations from the perspective of the nail. Adv Orthop 2016;2016:1967192.

8. Kumar VP, Satku K. Treatment and prevention of "hook nail" deformity with anatomic correlation. J Hand Surg Am 1993;18:617-20.

9. Kuret Z, Burger H, Vidmar G, et al. Adjustment to finger amputation and silicone finger prosthesis use. Disabil Rehabil 2019;41:1307-12.

10. Lee KJ, Kim YW, Kim JS, et al. Nail bed defect reconstruction using a thenar fascial flap and subsequent nail bed grafting. Arch Plast Surg 2019;46:57-62.

11. Adani R, Marcoccio I, Tarallo L. Nail lengthening and fingertip amputations. Plast Reconstr Surg 2003;112:1287-94.

12. Rai A, Jha MK, Makhija LK, et al. An algorithmic approach to posttraumatic nail deformities based on anatomical classification. J Plast Reconstr Aesthet Surg 2014;67:540-7. 